\title{
Sesquialtera in the Colombian Bambuco: Perception and Estimation of Beat and Meter
}

\section{Supplementary Material}

1.1 Trio Dataset Availability

\begin{tabular}{|c|c|c|}
\hline Title & Link & Last accessed \\
\hline Bambuco en Bm & https://youtu.be/_JOXozDZQPw & June 8, 2021 \\
\hline Cuadro de bambuco & https: //wWw. youtube. com/watch?v=6ZsiJZIsJt8 & June 8,2021 \\
\hline El parrandista & https://youtu.be/VSSeKVssqGo & June 8,2021 \\
\hline El republicano & https: //www. youtube. com/watch?v=cVoG6gQ0GSc & June 8, 2021 \\
\hline Fusagasugueño & $\begin{array}{l}\text { https: //www. youtube. com/watch?v=yDzEAr1b4EU\& } \\
\text { list=PLBdzXBcUlXUTm1JKlj1HrsLIy3g5-RCk1\&index=51 }\end{array}$ & June 8, 2021 \\
\hline Garrapatica & https: //www. youtube. com/watch?v=oyCyRnzekdE & June 8, 2021 \\
\hline Gloria Beatriz & https: //youtu.be/ezTS1aCPXC4 & June 8,2021 \\
\hline Los doce & $\begin{array}{l}\text { https: //www. youtube. com/watch?v=0-7cr9xbCZM\&t= } \\
1777 \mathrm{~s}\end{array}$ & June 8,2021 \\
\hline Nueva Colombia & https://youtu.be/-yD-Ow17LIM?t=3125 & June 8, 2021 \\
\hline Pa' Juancho & https: //www. youtube. com/watch?v=Vo3olIHVgtc & June 8,2021 \\
\hline Pilarica & https://youtu.be/yDzEAr1b4EU?t=1144 & June 8,2021 \\
\hline Verónica & https://www. youtube. com/watch?v=fgT3tcIH16g & June 8, 2021 \\
\hline
\end{tabular}

Table 1: Trio dataset. The title, url and the date each video was last accessed are included. 